\title{
Efecto de un protocolo de entrenamiento de fuerza con característica socializadora sobre el ángulo cráneovertebral en adultos mayores
}

\author{
Effect of a strength training protocol with a socializing characteristic on \\ the craniovertebral angle in older adults
}

\author{
* Sebastián Andrés Astorga Verdugo, ** Soledad Patricia González Silva, \\ *Fernanda Borges Silva, *Aldo Rodrigo Martínez Araya \& *Germán Rojas Cabezas
}

\begin{abstract}
Astorga, S., González, S., Borges, F., Martínez, A., \& Rojas, G. (2020). Efecto de un protocolo de entrenamiento de fuerza con característica socializadora sobre el ángulo cráneovertebral en adultos mayores. Revista Ciencias de la Actividad Física UCM, N²1(2), julio-diciembre, 1-9. DOI: http://doi.org/10.29035/rcaf.21.2.8
\end{abstract}

\section{RESUMEN}

Objetivo: El propósito del estudio fue evaluar el efecto de un protocolo de entrenamiento de fuerza con característica socializadora sobre el ángulo cráneovertebral en adultos mayores.

Material y métodos: El presente estudio tiene un diseño experimental, longitudinal, basado en la medición del ángulo cráneovertebral previo y posterior a la aplicación de un protocolo de entrenamiento de fuerza con característica socializadora de 4 meses. Se estudió a 3 grupos de 44 participantes elegidos al azar: dos grupos experimentales y un grupo control. A un grupo experimental se le realizó un protocolo de entrenamiento de fuerza convencional, al otro grupo experimental se le realizó el protocolo de entrenamiento de fuerza con característica socializadora y al grupo control, no se le aplicó entrenamiento. Los 132 participantes cumplieron los criterios de inclusión y exclusión: adultos mayores > 60 años, ángulo cráneovertebral < 50 grados, sin patologías de columna vertebral, reumatológicas, neurológicas y sistémicas.

Resultados: Hubo cambios estadísticamente significativos en el aumento del ángulo cráneovertebral en el grupo que realizó entrenamiento de fuerza convencional y en el grupo que realizó entrenamiento de fuerza con característica socializadora $(P<0.05)$.

Conclusión: El protocolo de entrenamiento de fuerza con característica socializadora aumentó en promedio 14,6 grados el ángulo cráneovertebral y fue un 21\% más efectivo que el grupo que realizó entrenamiento de fuerza convencional en el aumento del ángulo cráneovertebral en adultos mayores.

Palabras clave: entrenamiento de fuerza, ángulo cráneovertebral, adultos mayores.

* Universidad Autónoma de Chile, Chile.

** Universidad Católica de Murcia, España. 
Astorga, S., González, S., Borges, F., Martínez, A., \& Rojas, G. (2020). Efecto de un protocolo de entrenamiento de fuerza con característica socializadora sobre el ángulo cráneovertebral en adultos mayores. Revista Ciencias de la Actividad Física UCM, № 21(2), julio-diciembre, 1-9.

DOI: http://doi.org/10.29035/rcaf.21.2.8

\section{ABSTRACT}

Objective: Evaluate the effect of a strength training protocol with a socializing characteristic on the craniovertebral angle in older adults.

Material and methods: The present study has an experimental, longitudinal design, based on the measurement of the craniovertebral angle before and after the application of a 4 month long strength training protocol with a socializing characteristic. Three groups of 44 randomly chosen participants were carried out: two experimental groups and a control group. A conventional strength training protocol was performed in one experimental group, the strength training protocol with a socializing characteristic was performed in the other experimental group, and no training was applied to the control group. The 132 participants fulfilled the inclusion and exclusion criteria: older adults > 60 years, craniovertebral angle $<50$ degrees, without spinal, rheumatic, neurological and systemic pathologies.

Results: There were statistically significant changes in the increase of the craniovertebral angle in the group that performed conventional strength training and in the group that performed strength training with a socializing characteristic $(P<0.05)$.

Conclusion: The strength training protocol with socializing characteristic increased the craniovertebral angle by an average of 14.6 degrees and was $21 \%$ more effective than the group that performed conventional resistance training in increasing the craniovertebral angle in older adults.

Key words: strength training, craniovertebral angle, older adults.

\section{Introducción}

Los adultos mayores presentan cambios posturales asociados a la edad y a diversas posturas viciosas que adoptan durante su ciclo vital. Dentro de las posturas viciosas más frecuentes se encuentra la disminución del ángulo cráneovertebral en el plano horizontal (Kim, Kim \& Son, 2018). El ángulo cráneovertebral, se mide entre la intersección de la línea horizontal que pasa por el proceso espinoso de la vértebra cervical C7 y la línea articular del punto medio del tragus auricular de la oreja (Szczygieł, Sieradzki, Masłoń, Golec, Czechowska, Węglarz, ... Golec, 2019). El ángulo cráneovertebral se clasifica como normal $>50$ grados y un ángulo $<50$ grados se considera con anteposición de cabeza y cuello (Mani, Sharma, Omar, Ahmad, Muniandy \& Singh, 2017, Salahzadeh, Maroufi, Ahmadi, Behtash, Razmjoo, Gohari \& Parnianpour, 2014). La disminución del ángulo cráneovertebral, se asocia con un desplazamiento anterior del centro de gravedad que se caracteriza por un desplazamiento promedio de 5 centímetros en referencia al eje del cuerpo, produciendo aumento de la carga compresiva sobre las vértebras cervicales, afectando la tensión cervical posterior del tejido miofascial, favoreciendo dolores de cabeza y cuello (Szczygieł et al., 2019). Además, produce hiperextensión de la columna cervical superior (C1-C3), generado por acortamiento del trapecio superior, extensores cervicales (suboccipitales, semiespinal, esplenio, esternocleidomastoideo y elevador de la escápula) y una flexión de la columna cervical inferior (C4-C7).

El entrenamiento de fuerza (circuitos) a largo plazo es el tratamiento estándar para el aumento del ángulo cráneovertebral (Fathollahnejad, Letafatkar \& Hadadnezhad, 2019). Se han descrito efectivos protocolos de entrenamiento de fuerza (convencional), que se basan principalmente en la activación de los flexores cráneocervicales, activación de los retractores escapulares y la flexibilidad de la musculatura cervical posterior y de la musculatura bilateral de pectoral (Sheikhhoseini, Shahrbanian, Sayyadi \& O'Sullivan, 2018). Sin 
Astorga, S., González, S., Borges, F., Martínez, A., \& Rojas, G. (2020). Efecto de un protocolo de entrenamiento de fuerza con característica socializadora sobre el ángulo cráneovertebral en adultos mayores. Revista Ciencias de la Actividad Física UCM, № 21(2), julio-diciembre, 1-9.

DOI: http://doi.org/10.29035/rcaf.21.2.8

embargo, dentro de los inconvenientes para el éxito de los protocolos de entrenamientos músculo esqueléticos se encuentra la baja adherencia de los pacientes adultos mayores a sus terapias kinesiológicas (terapia manual, ejercicio físico y educación), principalmente por desmotivación (Jack, Mairi, Klaber \& Gardiner, 2010, Shiravi, Letafatkar, Bertozzi, Paolo \& Khaleghi, 2019).

La socialización entre los adultos mayores a través de actividades grupales y entretenidas, permite generar lazos de afectividad entre los participantes y facilita la adherencia a los protocolos de entrenamiento músculo esquelético, permitiendo que el entrenamiento se logre realizar a largo plazo (Morse, Xiong, Ramirez, Anne, Barish \& Lindquist, 2018). Los protocolos de entrenamiento de fuerza convencionales (circuitos) habitualmente se enfocan al fortalecimiento de grupos musculares específicos y se basan en sesiones individuales y personalizadas, facilitando el retiro prematuro de los participantes a sus protocolos de rehabilitación (Donghoon, 2019, Harman, Hubley \& Butler, 2010, Wickstrom, Oakley \& Harrison, 2017).

El objetivo del estudio fue evaluar el efecto de un protocolo de entrenamiento de fuerza con característica socializadora sobre el ángulo cráneovertebral en adultos mayores.

\section{MATERIALES Y MÉTODOS}

La presente investigación fue aprobada por el Comité de Ética de la Universidad Católica de Murcia, España (código CE061918).

La investigación presentó un diseño experimental, longitudinal y fue aplicado al Club del Adulto Mayor (CAM), perteneciente a la llustre Municipalidad de Talca, Chile.
Para la recogida de datos se revisaron 240 antecedentes generales de salud, de los cuales 132 participantes cumplieron los criterios de inclusión; adultos mayores > 60 años, ángulo cráneovertebral < 50 grados y exclusión; sin presencia de patologías traumáticas de columna vertebral, reumatológicas, neurológicas y sistémicas. El estudio fue realizado en el segundo semestre del año 2019.

\section{Participantes}

Los participantes se dividieron al azar mediante un muestreo probabilístico de tipo aleatorio simple en tres grupos de 44 participantes: un grupo control (pasivo) y dos grupos experimentales (grupo entrenamiento de fuerza convencional y grupo entrenamiento de fuerza con característica socializadora). Se utilizó la técnica de enmascaramiento doble ciego.

\section{Grupos de investigación (Tabla 1)}

1. Grupo de entrenamiento de fuerza convencional: se le realizó evaluación inicial (medición de ángulo cráneovertebral), entrenamiento de fuerza convencional por 4 meses y evaluación final (medición de ángulo cráneovertebral).

2. Grupo 2: grupo de entrenamiento de fuerza con característica socializadora. Se le realizó evaluación inicial (medición de ángulo cráneovertebral), entrenamiento de fuerza convencional con característica socializadora por 4 meses y evaluación final (medición de ángulo craáeovertebral).

3. Grupo control: se le realizó evaluación inicial y final (medición de ángulo cráneovertebral), sin realización de entrenamiento. 
Astorga, S., González, S., Borges, F., Martínez, A., \& Rojas, G. (2020). Efecto de un protocolo de entrenamiento de fuerza con característica socializadora sobre el ángulo cráneovertebral en adultos mayores. Revista Ciencias de la Actividad Física UCM, № 21(2), julio-diciembre, 1-9. DOI: http://doi.org/10.29035/rcaf.21.2.8

Tabla 1

Grupos de investigación ( $n=132)$.

\begin{tabular}{lccc} 
& $\begin{array}{c}\text { E. de fuerza convencional } \\
(\mathrm{n}=44)\end{array}$ & $\begin{array}{c}\text { E. de fuerza con socialización } \\
(\mathrm{n}=44)\end{array}$ & $\begin{array}{c}\text { Control } \\
(\mathrm{n}=44)\end{array}$ \\
\hline Evaluación inicial & $\times$ & $\times$ & $\times$ \\
E. de fuerza & $\times$ & $\times$ & \\
Socialización & & $\times$ & $\times$ \\
Evaluación final & $\times$ & & \\
\hline
\end{tabular}

Leyenda: E.: Entrenamiento.

\section{MEDICIÓN DEL ÁNGULO CRÁNEOVERTEBRAL}

Se realizó medición del ángulo cráneovertebral inicial (pre entrenamiento) y final (posterior a 5 días finalizados los protocolos de entrenamiento de 4 meses). La validez y confiabilidad del ángulo cráneovertebral ha sido investigado por diversos autores (Hanney, George, Kolber, Young, Salamh \& Cleland, 2014, Johnson \& Phty, 1998, Salahzadeh et al., 2014).

La medición del ángulo cráneovertebral se realizó mediante el software BTS Bioengineering, que es un sistema integrado de cuatro cámaras de alta resolución, en el laboratorio de biomecánica de la Universidad Autónoma de Chile, sede Talca. Para la evaluación cinemática en el plano sagital se utilizó un trípode a $265 \mathrm{~cm}$ de la pared, y se ajustó a la altura de los hombros. Se midió el ángulo cráneovertebral (se trazó una línea entre el tragus auricular y el proceso espinoso de C7 y se proyectó con eje horizontal)

Se les solicitó a los participantes que realizaran tres repeticiones de flexión y extensión cervical con los brazos cruzados en posición sedente y se le solicitó que vuelva a su posición cervical original. Se capturaron fotogramas en la posición final de las participantes después de 5 segundos. El promedio de las tres repeticiones es el ángulo cráneovertebral elegido.

\section{PROTOCOLO DE ENTRENAMIENTOS DE GRUPOS EXPERIMENTALES}

\section{Entrenamiento de fuerza convencional}

Cada participante realizó el ejercicio individualmente y se basó en circuitos de activación de flexores cráneocervicales y activación de retractores escapulares ( 3 series de 12 repeticiones) y elongaciones de musculatura cervical posterior y elongación bilateral de pectorales (3 repeticiones de 30 segundos cada uno). La duración promedio de la sesión fue de 40 minutos. Las sesiones se realizaron 2 veces por semana durante 4 meses.

\section{Entrenamiento de fuerza con característica socializadora}

Se realizaron los ejercicios grupalmente, se basaron en circuitos de activación de flexores cráneocervicales y retractores escapulares (3 series de 12 repeticiones) y elongaciones de musculatura cervical posterior y elongación bilateral de pectorales (3 repeticiones de 30 segundos cada uno) y se adicionaron actividades grupales de juegos (lanzamiento de dardos, juegos de memoria cognitiva, narración historias personales, etc.), estas actividades de juegos se modificaban cada dos semanas. La duración promedio de la sesión fue de 45 minutos. Las sesiones se realizaron 2 veces por semana durante 4 meses. 
Astorga, S., González, S., Borges, F., Martínez, A., \& Rojas, G. (2020). Efecto de un protocolo de entrenamiento de fuerza con característica socializadora sobre el ángulo cráneovertebral en adultos mayores. Revista Ciencias de la Actividad Física UCM, № 21(2), julio-diciembre, 1-9.

DOI: http://doi.org/10.29035/rcaf.21.2.8

\section{Análisis de datos}

Los datos se han analizado estadísticamente a través de Excel y SPSS versión 25. Se ha resguardado la confidencialidad de las historias clínicas y evaluaciones de los participantes.

Se realizaron pruebas de normalidad (Kolmogorov Smirnov, asimetría y curtosis y Q-Q plot), obteniendo una distribución normal. Se realizó prueba de ANOVA post hoc para la comparación de los 3 grupos y la prueba de regresión lineal para la relación entre el ángulo cráneovertebral y los grupos de investigación.

\section{RESULTADOS}

Se observa en el perfil general de los 132 participantes que el 16,3\% fue de sexo masculino y el $83,7 \%$ fue de sexo femenino, la edad promedio fue de 71 años, el detalle por grupo se observa en la Tabla 2.

Tabla 2

Características de la muestra (n=132).

\begin{tabular}{|c|c|c|c|}
\hline \multicolumn{4}{|c|}{ Sexo (\%) } \\
\hline Grupos & Masculino & Femenino & Edad (DE) \\
\hline Control $(n=44)$ & 36 & 64 & $71(6.1)$ \\
\hline $\operatorname{EFC}(n=44)$ & 9 & 91 & $71(7.1)$ \\
\hline EFS $(n=44)$ & 4 & 96 & $71(5.6)$ \\
\hline
\end{tabular}

Leyenda: EFC: Entrenamiento de fuerza convencional, EFS: Entrenamiento de fuerza con característica socializadora, DE: desviación estándar.

Tabla 3

Ángulo cráneovertebral pre y post entrenamiento (ANOVA).

\begin{tabular}{lccc}
\hline Ángulo cráneovertebral previo al entrenamiento & & \\
\hline Grupos & Promedio (grados) & DE & Sig. (P<0.05) \\
Control & 38.7 & 4.0 & \\
EFC & 38.9 & 4.7 & \\
EFS & 33.6 & 4.4 & 0.35 \\
\hline Ángulo cráneovertebral posterior al entrenamiento & & $0.00^{*}$ \\
\hline Control & 37.5 & 4.2 & $0,00^{*}$ \\
\hline EFC & 48.3 & 4.6 & 5.6 \\
EFS & 48.2 & 5.6 & \\
\hline
\end{tabular}

Leyenda: EFC: Entrenamiento de fuerza convencional, EFS: Entrenamiento de fuerza con característica socializadora, DE: desviación estándar.

El grupo experimental que realizó el protocolo de entrenamiento de fuerza con característica socializadora obtuvo un promedio de 14.6 grados de aumento del ángulo cráneovertebral al finalizar los 4 meses de entrenamiento.

El grupo experimental que realizó el protocolo de entrenamiento de fuerza convencional obtuvo un promedio de 9,4 grados de aumento del ángulo cráneovertebral al finalizar los 4 meses. El grupo control promedio disminuyó 0,6 grados el ángulo cráneovertebral al finalizar los 4 meses.

Los dos grupos experimentales obtuvieron cambios estadísticamente significativos $(P<0.05)$ en el aumento del ángulo cráneovertebral. 
Astorga, S., González, S., Borges, F., Martínez, A., \& Rojas, G. (2020). Efecto de un protocolo de entrenamiento de fuerza con característica socializadora sobre el ángulo cráneovertebral en adultos mayores. Revista Ciencias de la Actividad Física UCM, № 21(2), julio-diciembre, 1-9.

DOI: http://doi.org/10.29035/rcaf.21.2.8

Tabla 4

Relación entre ángulo cráneovertebral y grupos de investigación.

\begin{tabular}{lccc}
\hline & \multicolumn{2}{c}{ Coeficientes no estandarizados } & \\
\hline Grupos & $\mathrm{R}^{2}$ & B estándar & Error \\
Control & 0.83 & 0.95 & 0.66 \\
EFC & 0.36 & 0.21 & 0.17 \\
EFS & 0.40 & 0.25 & 0.19 \\
\hline
\end{tabular}

Variable dependiente: Ángulo cráneovertebral post

$0,258-0,213 / 0,213=0,21(21 \%)$

El modelo explica que el grupo que recibió el protocolo de entrenamiento de fuerza con característica socializadora fue un $21 \%$ más efectivo que el grupo que recibió el entrenamiento de fuerza convencional.

\section{DISCUSIÓN}

Los principales hallazgos indicaron que la característica socializadora en el entrenamiento de fuerza fue el factor más relevante para el aumento del ángulo cráneovertebral. Si bien el grupo que realizó entrenamiento de fuerza sin socialización obtuvo cambios significativos, estos fueron bastante inferiores. Mientras que el grupo control no tuvo cambios significativos. El aumento del ángulo cráneovertebral en el grupo que realizó entrenamiento con característica socializadora, podría explicarse por la producción de lazos afectivos entre los participantes y las actividades entretenidas realizadas semanalmente (Morse et al., 2018). Estos factores influyen directamente en la motivación de los adultos mayores para asistir de manera regular a participar en los protocolos de entrenamiento de fuerza músculo esquelético. Por lo tanto, podría ser relevante la incorporación de actividades grupales, las actividades entretenidas y la socialización en los protocolos de entrenamiento musculo esquelético en los adultos mayores. Nuestros hallazgos revelaron que la socialización era la variable más fuertemente asociada a los cambios en el ángulo cráneovertebral. En ese contexto, cabe señalar que el cambio en el ángulo cráneovertebral es un requisito biomecánico importante para mantener el equilibrio postural (Haider, Luger, Kapan, Titze \% Lackinger, 2016)

Según la literatura, los entrenamientos de fuerza a largo plazo para el aumento del ángulo cráneovertebral son efectivos (Sheikhhoseini et al., 2018); éstos se enfocan en la activación muscular de largo del cuello, largo de la cabeza, romboides y flexibilidad de músculos cervicales posteriores y elongación bilateral de pectorales, sin embargo, requieren del compromiso y adherencia de los participantes para el logro de resultados. Los protocolos estándar de entrenamiento de fuerza (circuitos) utilizados para el aumento del ángulo cráneovertebral incorporan el ejercicio físico de manera personalizada sin integración social por parte de los participantes (Mahmoud, Hassan, Abdelmajeed, Moustafa \& Silva, 2019). Nuestra investigación, se basó en innovar a través de la incorporación de actividades entretenidas para lograr mayor compromiso y motivación de los participantes.

Se ha investigado que los adultos mayores presentan diversas características especiales que influyen en la baja adherencia a los protocolos músculo esqueléticos a largo plazo, entre ellos destacan: desmotivación, disminución de la interacción social, depresión y el mayor riesgo de caídas (Kojima, Taniguchi, Kitamura \& Fujiwara, 2020), por ende, la 
Astorga, S., González, S., Borges, F., Martínez, A., \& Rojas, G. (2020). Efecto de un protocolo de entrenamiento de fuerza con característica socializadora sobre el ángulo cráneovertebral en adultos mayores. Revista Ciencias de la Actividad Física UCM, № 21(2), julio-diciembre, 1-9.

DOI: http://doi.org/10.29035/rcaf.21.2.8

socialización como parte del protocolo de entrenamiento puede influir positivamente en el cambio del ángulo cráneovertebral a largo plazo (Jessen \& Lund, 2017). Los resultados obtenidos en nuestra investigación pueden ser justificados por la ayuda en el factor motivacional favoreciendo la asistencia de los adultos mayores al programa de entrenamiento a largo plazo (Gupta, Aggarwal, Gupta, Gupta, \& Gupta, 2013, Harman et al., 2010).

Según la literatura el promedio de aumento del ángulo cráneovertebral de los programas de entrenamiento de fuerza convencionales es 4,5 grados, mientras que en nuestra investigación aumentó 14,6 grados (Sheikhhoseini et al., 2018), demostrando la importancia de innovar en el tipo de terapia músculoesqueletica que se realiza.

Sería interesante para futuras investigaciones evaluar el porcentaje de adherencia al tratamiento de los adultos mayores.

\section{CONCLUSIÓN}

El protocolo de entrenamiento de fuerza con característica socializadora a largo plazo aumentó en promedio 14,6 grados el ángulo cráneovertebral en los adultos mayores y fue un $21 \%$ más efectivo versus el protocolo de entrenamiento de fuerza convencional.

\section{Conflicto de intereses}

Los autores declaran no tener ningún conflicto de intereses.

\section{Financiación}

La presente investigación no ha recibido ayudas específicas provenientes de agencias del sector público, sector comercial o entidades sin ánimo de lucro.

\section{REFERENCIAS BIBLIOGRÁFICAS}

Donghoon Kang, T. O. (2019). Comparison of the muscle activity in the normal and forward head postures based on the pressure level during cranio-cervical flexion exercises. The Journal of Korean Physical Therapy 37(1), 1-6. DOI: https://doi.org/10.18857/jkpt.2019.31.1.1

Fathollahnejad, K., Letafatkar, A., \& Hadadnezhad, M. (2019). The effect of manual therapy and stabilizing exercises on forward head and rounded shoulder postures: A six-week intervention with a one-month follow-up study. BMC Musculoskeletal Disorders, 20(1), 1-8. DOI: https://doi.org/10.1186/s12891$019-2438-y$

Gupta, B., Aggarwal, S., Gupta, B., Gupta, M., \& Gupta, N. (2013). Effect of deep cervical flexor training vs. conventional isometric training on forward head posture, pain, neck disability index in dentists suffering from chronic neck pain. Journal of Clinical and Diagnostic Research, 7(10), 2261-2264. DOI: https://doi.org/10.7860/JCDR/2013/6072.34 87

Haider, S., Luger, E., Kapan, A., Titze, S., \& Lackinger C. (2016). Associations between daily physical activity Associations between daily physical activity, handgrip strength, muscle mass, physical performance and quality of life in prefrail and frail community-dweIling older adults. Qual Life Res (25)31293138. DOl: http://doi.org/10.1007/s11136-0161349-8

Hanney, W. J., George, S. Z., Kolber, M. J., Young, I., Salamh, P. A., \& Cleland, J. A. (2014). Interrater reliability of select physical examination procedures in patients with neck pain. Physiotherapy Theory and Practice, 30(5), 345-352.

DOI: https://doi.org/10.3109/09593985.2013.8702 67 
Astorga, S., González, S., Borges, F., Martínez, A., \& Rojas, G. (2020). Efecto de un protocolo de entrenamiento de fuerza con característica socializadora sobre el ángulo cráneovertebral en adultos mayores. Revista Ciencias de la Actividad Física UCM, № 21(2), julio-diciembre, 1-9.

DOI: http://doi.org/10.29035/rcaf.21.2.8

Harman, K., Hubley-Kozey, C. L., \& Butler, H. (2010). Effectiveness of an Exercise Program to Improve Forward Head Posture in Normal Adults: A Randomized, Controlled 10-Week Trial. Journal of Manual \& Manipulative Therapy, 13(3), 163-176. DOI: https://doi.org/10.1179/10669810579082488 8

Jack, K., Mairi, S., Klaber, J., \& Gardiner, E. (2010). Barriers to treatment adherence in physiotherapy outpatient clinics: a systematic review. Manual Therapy, 75(3), 220-228. DOI: https://doi.org/10.1016/j.math.2009.12.004

Jessen, J. D., \& Lund, H. H. (2017). Study protocol: effect of playful training on functional abilities of older adults - a randomized controlled trial. BMC Geriatrics, 17(7), 1-7. DOI: https://doi.org/10.1186/s12877-017-0416-5

Johnson, G., \& Phty, D. (1998). The correlation between surface measurement of head and neck posture and the anatomic position of upper cervical vertebrae. Spine, 23(8), 921927. DOI: 10.1097/00007632-19980415000015

Kim, D. H., Kim, C. J., \& Son, S. M. (2018). Article history: Neck Pain in Adults with Forward Head Posture: Effects of Craniovertebral Angle and Cervical Range of Motion Osong Public Health and Research Perspectives. Public Health Res Perspect, 9(6), 309-313. DOl: https://doi.org/10.24171/j.phrp.2018.9.6.04

Kojima, G., Taniguchi, Y., Kitamura, A., \& Fujiwara, Y. (2020). Is living alone a risk factor of frailty? A systematic review and meta-analysis. Ageing Research Reviews, (59), 101048. DOl: https://doi.org/10.1016/j.arr.2020.101048

Mahmoud, N. F., Hassan, K. A., Abdelmajeed, S. F., Moustafa, I. M., \& Silva, A. G. (2019). The Relationship Between Forward Head Posture and Neck Pain: a Systematic Review and Meta-Analysis. Current Reviews in
Musculoskeletal Medicine 12(4), 562-577.

DOI: $\quad$ https://doi.org/10.1007/s12178-01909594-y

Mani, S., Sharma, S., Omar, B., Ahmad, K., Muniandy, Y., \& Singh, D. K. A. (2017). Quantitative measurements of forward head posture in a clinical settings: a technical feasibility study. European Journal of Physiotherapy, 19(3), 119-123. DOI: https://doi.org/10.1080/21679169.2017.12968 88

Morse, L. A., Xiong, L., Ramirez-Zohfeld, V., Anne, S., Barish, B., \& Lindquist, L. A. (2018). Humor doesn't retire: Improvisation as a healthpromoting intervention for older adults. Archives of Gerontology and Geriatrics, (75) 1-5. DOl: https://doi.org/10.1016/j.archger.2017.10.013

Salahzadeh, Z., Maroufi, N., Ahmadi, A., Behtash, H., Razmjoo, A., Gohari, M., \& Parnianpour, M. (2014). Assessment of forward head posture in females: Observational and photogrammetry methods. Journal of Back and Musculoskeletal Rehabilitation, 27(2), 131-139. DOl: https://doi.org/10.3233/BMR130426

Sheikhhoseini, R., Shahrbanian, S., Sayyadi, P., \& O'Sullivan, K. (2018). Effectiveness of Therapeutic Exercise on Forward Head Posture: A Systematic Review and Meta-analysis. Journal of Manipulative and Physiological Therapeutics, 47(6), 530-539. DOI: https://doi.org/10.1016/j.jmpt.2018.02.002

Shiravi, S., Letafatkar, A., Bertozzi, L., Paolo, P., \& Khaleghi Tazji, M. (2019). Efficacy of Abdominal Control Feedback and Scapula Stabilization Exercises in Participants With Forward Head, Round Shoulder Postures and Neck Movement Impairment. Sports Health: A Multidisciplinary Approach, 17(3), 272-279. DOl:

https://doi.org/10.1177/1941738119835223 
Astorga, S., González, S., Borges, F., Martínez, A., \& Rojas, G. (2020). Efecto de un protocolo de entrenamiento de fuerza con característica socializadora sobre el ángulo cráneovertebral en adultos mayores. Revista Ciencias de la Actividad Física UCM, № 21(2), julio-diciembre, 1-9.

DOI: http://doi.org/10.29035/rcaf.21.2.8

Szczygieł, E., Sieradzki, B., Masłoń, A., Golec, J., Czechowska, D., Węglarz, K., ... Golec, E. (2019). Assessing the impact of certain exercises on the spatial head posture. International Journal of Occupational Medicine and Environmental Health, 32(1), 43-51. DOI: https://doi.org/10.13075/ijomeh.1896.01293
Wickstrom, B. M., Oakley, P. A., \& Harrison, D. E. (2017). Non-surgical relief of cervical radiculopathy through reduction of forward head posture and restoration of cervical lordosis: a case report. Journal of Physical Therapy Science, 29(8), 1472-1474. DOl: https://doi.org/10.1589/jpts.29.1472

\section{Dirección para correspondencia}

Mg. Sebastián Andrés Astorga Verdugo

Universidad Autónoma de Chile, Chile.

Dirección postal: 5 poniente 1670, Talca, Chile.

ORCID: http://orcid.org/0000-0002-8672-4987

Contacto:

sastorgav@uautonoma.cl

Recibido: 27-05-2020

Aceptado: 26-11-2020 